\title{
INTEGRATED APPROACH TO ECG SIGNAL PROCESSING
}

\author{
Manpreet Kaur ${ }^{1}$, Ubhi J.S. ${ }^{2}$, Birmohan Singh ${ }^{3}$, Seema ${ }^{4}$ \\ ${ }^{1}$ Department of EIE, ${ }^{2,4}$ Department of ECE, \\ ${ }^{4}$ Department of CSE, Sant Longowal Institute of Engineering and Technology, Longowal (Pb), India - 148106 \\ Email: 1aneja_mpk@yahoo.com, ${ }^{2}$ js_ubhi@yahoo.com, ${ }^{3}$ birmohans@gmail.com, ${ }^{4}$ seemagoyal.2009@gmail.com
}

\begin{abstract}
The Electrocardiogram (ECG) Signal is a graphical representation of the electromechanical activity of the cardiac system. It is one of the most important physiological parameter, which is being extensively used for knowing the state of cardiac patients. While taking ECG signals, the noises get introduced. It leads to the interference of these noises to important cardiac information. In order to get rid of these noises, an appropriate filter must be designed. This paper provides the design of cascading of IIR Bandpass Zero phase (BP-ZP) Elliptic filter, median filter and Elliptic notch filter. It helps to reduce Low frequency artifacts, High frequency artifacts, Baseline wander and Power line Interference to large extent. This reduction is shown by calculating Power Spectral Density (PSD) of signal before and after implementing above mentioned filters on ECG signal. The results have been concluded using Matlab and MIT-BIH Arrhythmia database.
\end{abstract}

Key words: ECG, Noises, IIR, PSD, bandpass

\section{INTRODUCTION}

Heart diseases, which are one of the death reasons, are among the important problems in this century. According to latest survey, $60 \%$ of the patients die due to Heart problems. Today, in 2010, 10 crore people are suffering from heart diseases in India. Based on the 2001 Census, 5 crore people were affected by heart diseases. Many individuals remain unaware of the symptoms of heart attack or dismiss possible symptoms as being unrelated or not important enough to visit a doctor. Early diagnosis and medical treatment of heart diseases can prevent sudden death of the patient. One of the ways to diagnose heart diseases is to use Electrocardiogram (ECG) signals [1]. First step to analyse the ECG signals is Filtration which is very important, so as to get the parameters of ECG signal clear without noise. Cleaned ECG signal gives full detailed information about the electrophysiology of the heart diseases and ischemic changes that may occur [2]. Fig 1 shows Normal ECG trace. Each heartbeat produces a set of P-QRS-T waves [3].

For reliable interpretation of real-time ECGs, computer based techniques based on digital signal processing of ECG waveform have been reported [4]. There are several techniques available to reduce the noises of ECG signal like FIR or IIR digital filters, adaptive method and wavelet transform thresholding method in Literature.

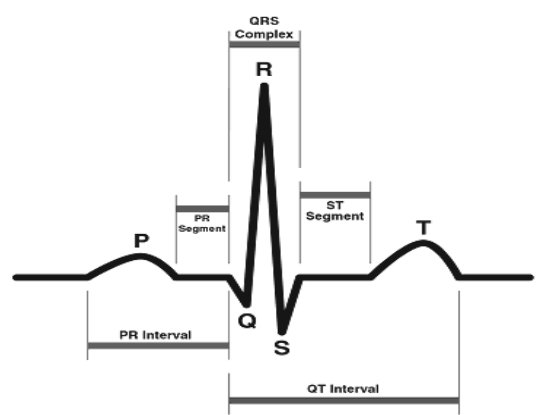

Fig. 1: Normal ECG wave form

\section{PROPOSED METHOD}

An ECG signal is normally corrupted with low frequency \& high frequency artifacts \& $50 / 60 \mathrm{~Hz}$ powerline Interference. The important information of ECG signal lies nearly in the range of $0.5 \mathrm{~Hz}-100 \mathrm{~Hz}$ [5]. So, the frequencies beyond that range can be easily eliminating using bandpass filter. In literature, paper [6] used both high pass and low pass filters to remove low frequency and high frequency noises respectively. But proposed method is designed by taking into consideration the computational complexity and time. Further to remove powerline interference this paper provides the cascading of median filter and elliptic notch filter.

\section{BANDPASS ZERO-PHASE IIR ELLIPTIC FILTER}

The elliptic filter is also called the Cauer filter. This filter has equiripple passband and stopband. For 
a given filter order, passband and stopband deviations, elliptic filters have the minimum transition bandwidth[7] and also satisfies the desired filter specifications with the lowest order among the other IIR filters. However, IIR filters have a nonlinear phase response which distorts meaningful components of the ECG waveform. To avoid this distortion, bidirectional filtering is performed, in which the signal is filtered in a forward direction over a selected window and then the same window is filtered in a reverse direction. A short window was selected so that the filter could be used for real time purposes [8].

In this paper the bandpass IIR elliptic filter of order 2 is designed with $360 \mathrm{~Hz}$ sampling frequency. The coefficients obtained in this filter are used to perform Zero phase filtering of ECG signal. The algorithm would be in the form [9]:

$$
\begin{aligned}
& y[n]=a_{0} x[n]-a_{1} x[n-1]-a_{2} x[n-2]-a_{3} x[n-3]-\ldots \\
& -b_{1} y[n-1]-b_{2} y[n-2]-b_{3} y[n-3]-\ldots
\end{aligned}
$$

Where $x[]$ is input signal, $y[]$ is output signal and a's and b's are coefficients.

This equation is called recursion equation. It will filter the signal by starting from first sample to last sample.

$$
\begin{aligned}
& y[n]=a_{0} x[n]-a_{1} x[n-1]-a_{2} x[n-2]-a_{3} x[n-3]-\ldots \\
& -b_{1} y[n-1]-b_{2} y[n-2]-b_{3} y[n-3]-\ldots
\end{aligned}
$$

It is reverse recursion equation. It will filter in opposite direction i.e. from last sample to first sample of input signal. This way, the delay of each frequency component is applied forwards and backwards in time and is therefore cancelled. Then the result has the following characteristics [8]:

- Zero-phase distortion

- A filter transfer function, which equals the squared magnitude of the original filter transfer function

- A filter order that is double the order of the filter

\section{MEDIAN FILTER}

Median filtering is a non-linear technique that applies a sliding window to the sequence. The median filter replaces the centre value in the window with the median value of all the points within the window. In computing this median the filter considers the signal to be 0 beyond the end points. Output has the same length as that of input.

For odd value of order,

$y(k)$ is the median of

$$
x(k-(n-1) / 2: k+(n-1) / 2)
$$

For even value of order,

$y(k)$ is the median of

$$
x(k-n / 2), x(k-(n / 2)+1) \ldots x(k+(n / 2)-1)
$$

where $y()$ is the output signal, $x()$ is input signal and $n$ is order of filter[8]. In this paper, the filter of order 6 is used.

\section{IIR ELLIPTIC NOTCH FILTER}

Power line interference is easily recognizable since the interfering voltage in the ECG may have frequency $50 / 60 \mathrm{~Hz}$ [10]. A well known method capable of reducing the power line interference is the use of a notch filter characterized by a unit gain at all frequencies except at notch frequency where the gain is zero [11]. A Notch filter is designed to remove a very specific, narrow band of frequencies. IIR notch filters offer very high attenuation with a low order [12]. The centre frequency of filter chosen to be exactly 60 $\mathrm{Hz}$ and bandwidth is $0.4 \mathrm{~Hz}$. In this design the second order filter is selected. The magnitude response of notch filter is shown in Fig 2.

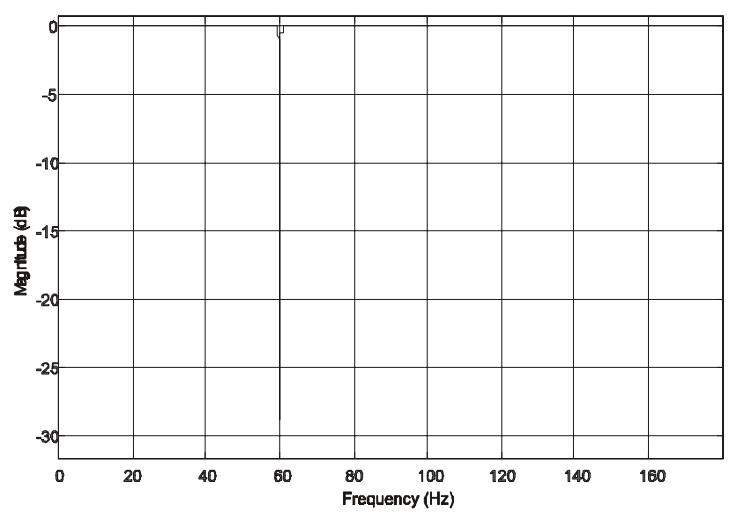

Fig. 2 Magnitude Response 


\section{RESULTING WAVEFORMS}

The results are obtained by taking the data of sample signal available in MIT-BIH database as shown in Fig. 3. The sequence of application of filters on original waveform is as follow: firstly, Zero-Phase Elliptic filter, secondly, median filter and at last elliptic notch filter. The waveforms obtained after applying filters in this sequence are as follows:

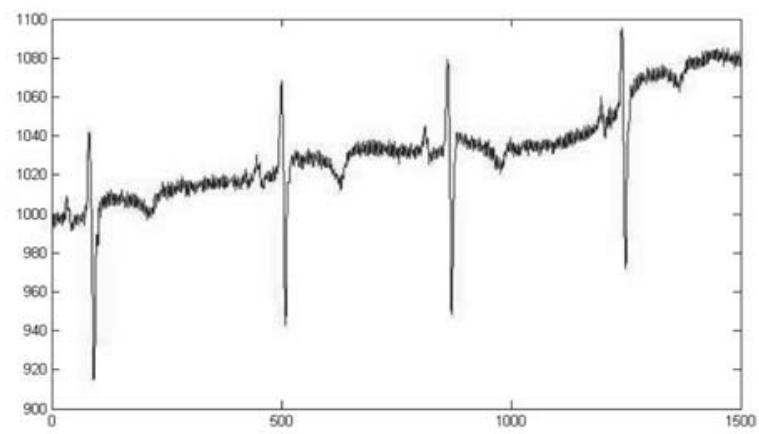

Fig. 3. Original ECG waveform

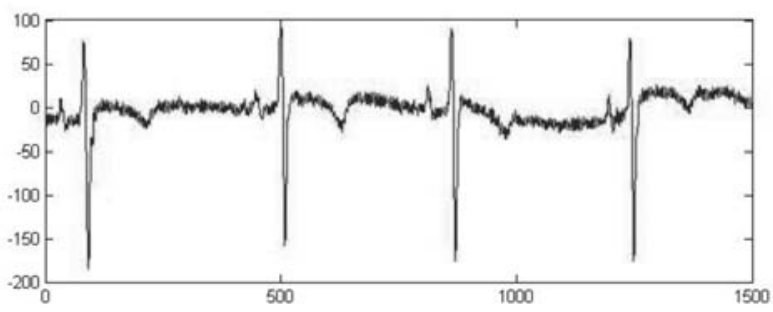

Fig. 4. Waveform after Bandpass Zero phase elliptic filter

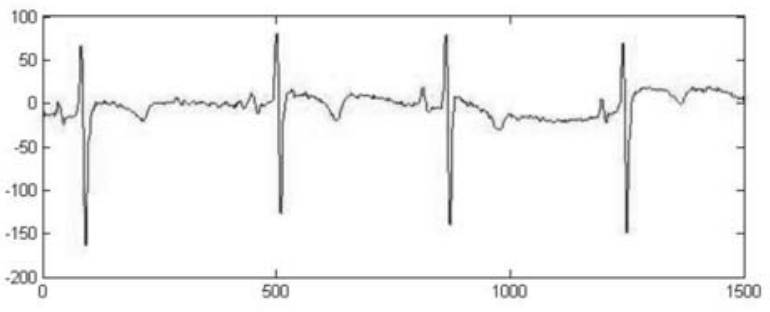

Fig. 5. Waveform after median filtering

\section{CALCULATION OF PARAMETERS}

Table 1 shows calculation of PSD and signal to noise ratio (SNR) of signal after applying the proposed series of filter \& Table 2 shows same parameters without using median filter in proposed cascaded filters.

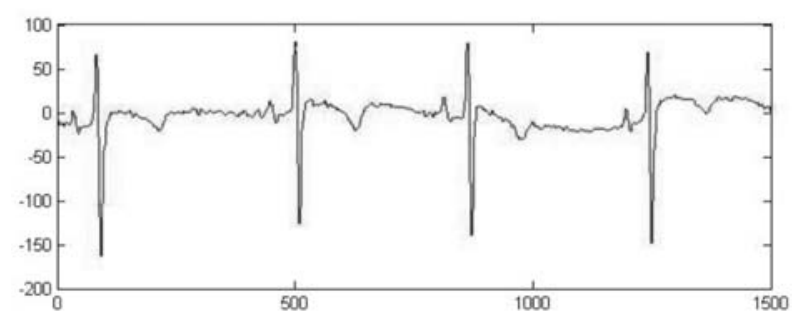

Fig. 6: Waveform after applying Notch filter

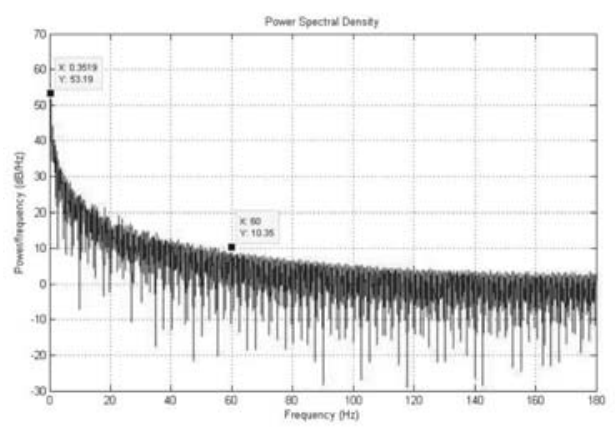

Fig. 7. PSD of original signal

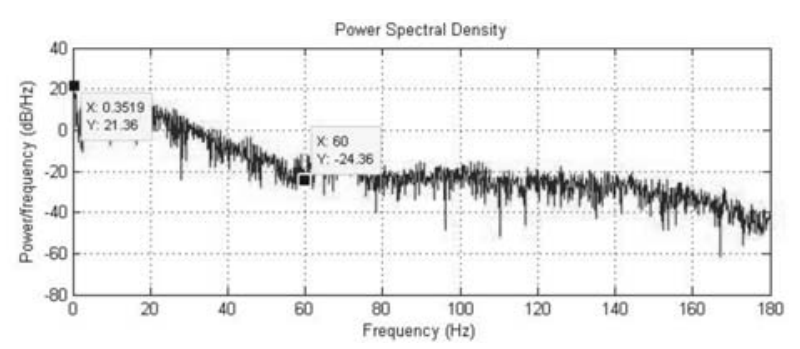

Fig. 8. PSD after applying proposed method

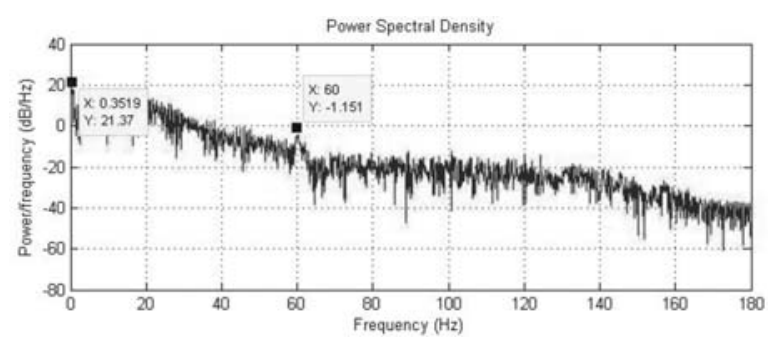

Fig. 9. PSD after notch filter directly (without median filtering)

\section{INTERPRETATION OF RESULTS}

It has observed that when BP-ZP elliptic filter is applied on original ECG signal, the low frequency and high frequency artifacts reduces effectively without producing any transients and distortion to waveform as 
Table1: Calculation of PSD and SNR after applying the Proposed cascaded filters

\begin{tabular}{|l|l|l|l|l|l|}
\hline Filter & $\begin{array}{l}\text { PSD at } 0.35 \mathrm{~Hz} \\
\text { (in dB/Hz) } \\
\text { before } \\
\text { filtration }\end{array}$ & $\begin{array}{l}\text { PSD at } 0.35 \mathrm{~Hz} \\
\text { (in dB/Hz) after } \\
\text { filtration }\end{array}$ & $\begin{array}{l}\text { PSD at } 60 \mathrm{~Hz} \\
\text { (in dB/Hz) } \\
\text { before } \\
\text { filtration }\end{array}$ & $\begin{array}{l}\text { PSD at 0.35Hz } \\
\text { (in dB/Hz) after } \\
\text { filtration }\end{array}$ & SNR (in dB) \\
\hline $\begin{array}{l}\text { BP-ZP Elliptic } \\
\text { filter }\end{array}$ & 53.19 & 21.37 & 10.35 & 13.17 & 0.92 \\
\hline Median filter & 21.37 & 21.36 & 13.17 & -9.10 & 1.12 \\
\hline $\begin{array}{l}\text { Elliptic Notch } \\
\text { filter }\end{array}$ & 21.36 & 21.36 & -9.10 & -24.36 & 1.12 \\
\hline
\end{tabular}

Table 2: Calculation of PSD and SNR without using median filter in Proposed cascaded filters

\begin{tabular}{|l|l|l|l|l|l|}
\hline Filter & $\begin{array}{l}\text { PSD at } 0.35 \mathrm{~Hz} \\
\text { (in dB/ } / \mathrm{Hz} \text { ) } \\
\text { before } \\
\text { filtration }\end{array}$ & $\begin{array}{l}\text { PSD at } 0.35 \mathrm{~Hz} \\
\text { (in } \mathrm{dB} / \mathrm{Hz} \text { ) } \\
\text { after filtration }\end{array}$ & $\begin{array}{l}\text { PSD at } 60 \mathrm{~Hz} \\
\text { (in dB/Hz) } \\
\text { before } \\
\text { filtration }\end{array}$ & $\begin{array}{l}\text { PSD at } 0.35 \mathrm{~Hz} \\
\text { (in dB/Hz) } \\
\text { after filtration }\end{array}$ & SNR (in dB) \\
\hline $\begin{array}{l}\text { BP-ZP Elliptic } \\
\text { filter }\end{array}$ & 53.19 & 21.37 & 10.35 & 13.17 & 0.92 \\
\hline $\begin{array}{l}\text { Elliptic Notch } \\
\text { filter }\end{array}$ & 21.37 & 21.37 & 13.17 & -1.15 & 0.90 \\
\hline
\end{tabular}

shown in Fig 4. The time of localization of QRS complex remains same as that of original wave. It also corrects the baseline drift. This reduction is shown mathematically by calculating the value of PSD below $0.5 \mathrm{~Hz}$. The value PSD reduces from $53.19 \mathrm{db} / \mathrm{Hz}$ to $21.37 \mathrm{db} / \mathrm{Hz}$ as shown in Table 1.

Bandpass filtering is followed by median filtering. This makes the appearance of ECG signal very much clean as shown in fig 5 and automatically reduces the value of PSD from $13.17 \mathrm{~dB} / \mathrm{Hz}$ to $-9.10 \mathrm{~dB} / \mathrm{Hz}$. Then after applying notch filter PSD further reduces to $-24.36 \mathrm{~dB} / \mathrm{Hz}$ as shown in Fig 8.The resulting waveform shown in Fig 6 free from any type of ringing effect and distortion. It has seen that when notch filter was applied directly without involving median filtering, the removal of Powerline interference was not so effective as shown in Fig 9.

There is also some improvement in signal to noise ratio by using median filtering as shown in Tables $1 \& 2$.

\section{CONCLUSIONS}

The filter proposed in this paper has removed various noises present in ECG signal significantly without any type of distortion. It makes the interpretation of ECG signal much easy. Due to IIR filters, it has fewer coefficients and so lesser computation time and complexity. So the above cascading of filters is more suitable and efficient for real time processing of ECG signals.

\section{REFERENCES}

[1] Y. Ozbay and B. Karlik, 2001, A Recognition Of ECG Arrhythmias Using Artificial Neural Networks, Proceedings 23rd Annual Conference, IEEE/EMBS Oct.25-28, 2001, Istanbul, TURKEY.

[2] Abdel-Rahman et al., 2010, ECG Signal Enhancement Using Wavelet Transform, WSEAS transactions on BIOLOGY and Biomedicine, Issue 2, Volume 7, April 2010, pp 62-72

[3] Wikipedia

[4] G. K. Singh, A. Sharma, S. Velusami, 2009, A Research Review on Analysis and Interpretation of Arrhythmias using ECG Signals, International Journal of Medical Sciences and Technology, 2009, 2(3), pp37-55.

[5] Ch. Renumadhavi et al., 2006, A New Approach for Evaluating SNR of ECG Signals and Its Implementation, Proceedings of the 6th WSEAS International Conference on Simulation, Modeling and 
Manpreet Kaur et al : Integrated Approach To Ecg Signal Processing...

Optimization, Lisbon, Portugal, September 22-24, 2006, pp 202-205

[6] M. S. Chavan, R.A.Aggarwala, M.D.Uplane, 2008, Interference reduction in ECG using digital FIR filters based on Rectangular window, WSEAS Transactions on Signal Processing, Issue 5, Volume 4, May 2008, pp340-49.

[7] S Salivahanan et al., Digital Signal processing, Tata McGraw Hill Publishing Company Limited.

[8] Matlab help MATLAB MATHWORKS. http://www mathworks.com/

[9] Steven W. smith, 1999, The Scientist and Engineer's guide to digital signal processing, second edition 1999, California Technical Publishing, San Diego, California.
[10] Mahesh S. Chavan, R.A.Aggarwala, M.D.Uplane, 2008, Design and implementation of Digital FIR Equiripple notch Filter on ECG signal for removal of Powerline Interference, WSEAS Transactions on Signal Processing, Issue 4, vol 4, pp. 221-230.

[11] Chien-Cheng Tseng and Su-Ling Lee, 2001, Elimination of Power Line Interference and Noise in Electrocardiogram Using Constrained Eigen filter, 0-7803-6685-9/01/2001 IEEE, pp II -405-408

[12] Tim Starr , 'Filtering A Noisy ECG Signal Using Digital Techniques' April $19,2005$. http://tzilla.is-ageek.com/school/ spring 2005/ee401/ design. 